\section{AR activity in treatment-naive prostate tumours}

New transcriptomic data from nearly 20,000 patients with localized prostate cancer reveal a previously uncharacterized subset of tumours that have low androgen receptor activity (ARA), biological and clinical similarities to metastatic castration-resistant prostate cancer (mCRPC) and specific sensitivities to systemic treatments.

The effects of varying ARA in men receiving systemic treatments for $\mathrm{mCRPC}$ are being extensively studied, but whether ARA is heterogeneous and has a role in outcomes in treatment-naive localized disease was unknown. In a new study, Spratt and colleagues first defined an ARA score and found that $\sim 10 \%$ of tumours across the tested cohorts were classified as having low ARA. Gene expression and immunohistochemistry analyses showed that tumours with low ARA were more likely to be negative for expression of ERG, ETS and SPINK and to have a basal phenotype than those with high ARA. Furthermore, gene expression of low ARA tumours was increased for immune signalling and neuroendocrine markers but decreased for DNA repair pathway markers.

Evaluating whether this different phenotype had prognostic relevance, the team found that men with low ARA tumours had an increased potential for metastatic disease according to Decipher scores. This finding was validated using clinical data, demonstrating that low ARA was significantly associated with an increased risk of developing metastatic disease (HR 2.61, $95 \% \mathrm{Cl} 1.22-5.60 ; P=0.01$ ) in a multivariate competing risks regression analysis.

Finally, the researchers found that tumours with low ARA were significantly less likely to be sensitive to androgen deprivation therapy (OR 0.41, 95\% Cl 0.21-0.80; $P=0.008$ ) and more likely to develop castration resistance than those with high ARA. Sensitivity scores of low ARA tumours were increased for platinum-based chemotherapy and poly(ADP-ribose) polymerase (PARP) inhibitors and decreased for docetaxel. Tumours with low ARA were also predicted to have increased potential for responses to radiotherapy.

Clemens Thoma

ORIGINAL ARTICLE Spratt, D. E. et al. Transcriptomic heterogeneity of androgen receptor (AR) activity defines a de novo low AR-active subclass in treatment naïve primary prostate cancer. Clin. Cancer Res. https://doi.org/10.1158/ 1078-0432.CCR-19-1587 (2019)

\title{
Gardnerella clade associated with sexual behaviours
}

Gardnerella vaginalis, which is detected in women both with and without bacterial vaginosis $(\mathrm{BV})$, is acquired through sexual activity and exists in both commensal and pathogenic clades, according to a new study in the Journal of Infectious Diseases.

BV (diagnosed as a Nugent score (NS) $>7$ ) is extremely common and responds poorly to treatment, meaning that many women experience a relapse within a year. Gardnerella is almost always present in women with BV and might have a role as a founder organism. However, as it is also present in many women without $\mathrm{BV}$, its precise role remains uncertain. The study aimed to investigate whether Gardnerella exists in both commensal and pathogenic clades and particularly focused on women who have sex with women (WSW) in whom $G$. vaginalis clade distribution data are lacking and to investigate its association with sexual behaviours.

Nonpregnant premenopausal women $(n=101)$ who reported a female sexual partner (FSP) in the past 18 months were enrolled. Overall, G. vaginalis was detected in 184/372 specimens (49\%) and varied by NS: $130 / 306$ with NS $=0-3(42 \%), 15 / 18$ with NS $=4-6$ $(83 \%)$ and $39 / 48$ specimens with Nugent-BV (81\%). G. vaginalis detection was associated with self-reported symptoms, NS $=4-6$ and Nugent-BV, as well as with Lactobacillusdeficient vaginal microbiota, smoking, history of penile-vaginal sex and frequent sex. Detection of clades 1, 2 and 3 and communities containing $\geq 2$ clades were associated with BV. Interestingly, specific clades were associated with specific sexual behaviours: clade 1 with an increased number of recent sexual partners and smoking, and clade 2 with penile-vaginal sex and sharing sex toys with female partners.

These data suggest that $G$. vaginalis is sexually transferred and that different clades might have different levels of pathogenicity. Thus, treating sexual partners could help to improve cure rates in women with BV.

Annette Fenner

ORIGINAL ARTICLE Plummer, E. L. et al. Gardnerella vaginalis clade distribution is associated with behavioural practices and Nugent score in women who have sex with women.J. Infect. Dis. https://doi.org/10.1093/infdis/jiz474 (2019)

\section{PROSTATE CANCER}

\section{UT-34: a promising new AR degrader}

UT-34, a newly discovered second-generation selective agent for the degradation of androgen receptor (SARD), degrades enzalutamidesensitive and enzalutamide-resistant androgen receptors (ARs) and AR splice variants (ARVs) in preclinical models of prostate cancer. This orally bioavailable SARD has promising drug-like characteristics and should progress to clinical development.

UT-34 was selected for further characterization from a library owing to its favourable degradation and antagonistic characteristics and pharmacokinetic properties compared with first-generation SARDs, whose pharmacokinetic properties were inappropriate for further development.

In vitro, UT-34 antagonized wild-type AR with a similar half-maximal inhibitory concentration $\left(\mathrm{IC}_{50}\right)$ to enzalutamide and had similar or better $\mathrm{IC}_{50} \mathrm{~s}$ in AR mutants. In LNCaP cells, UT-34 reduced $A R$ protein levels, unlike enzalutamide or bicalutamide, and also downregulated AR in enzalutamide-resistant MR49F cells.
In cross-reactivity experiments, UT-34 did not degrade other receptors.

Further analyses suggested that, to degrade AR, UT-34 requires the ubiquitin proteasome pathway. Moreover, UT-34 was found to bind to the activation function 1 region of AR. In LNCaP and LNCaP95 cells, treatment with UT-34 downregulated AR and AR-V7. Drug metabolism and pharmacokinetic assays showed that UT-34 is stable and efficacious. In vivo, oral treatment with UT-34 shrank the prostate more than treatment with enzalutamide. UT-34 treatment also decreased the growth of the enzalutamide-resistant, castration-resistant xenografts.

These data show that UT-34 is a promising SARD for the treatment of enzalutamide-resistant prostate cancer and warrants further clinical development.

Louise Stone

ORIGINAL ARTICLE Ponnusamy, S. et al. Orally-bioavailable androgen receptor degrader, potential next-generation therapeutic for enzalutamide-resistant prostate cancer. Clin. Cancer Res. https://doi.org/10.1158/1078-0432.CCR-19-1458 (2019) 